\title{
Mixed Phenotype Acute Leukemia with t(v;11q23.3); KMT2A Rearranged
}

National Cancer Institute

\section{Source}

National Cancer Institute. Mixed Phenotype Acute Leukemia with t(v;11q23.3); KMT2A

Rearranged. NCI Thesaurus. Code C82203.

A rare mixed phenotype acute leukemia in which the blasts also carry a translocation ( $v$; 11q23.3) involving the KMT2A gene. The prognosis is usually unfavorable. 